\title{
Effect of Multidimensional Exercise Program for Improving Balance in Traumatic Brain Injury Patients
}

\author{
Pranali Kakade ${ }^{1}$, Suraj B Kanase ${ }^{2}$ \\ ${ }^{1}$ Intern, Physiotherapy, Krishna College of Physiotherapy, Krishna Institute of Medical Sciences, Karad, \\ ${ }^{2}$ Associate Professor, Department of Neuroscience of physiotherapy, Krishna College of Physiotherapy, Krishna \\ Institute of Medical Sciences, Karad
}

\begin{abstract}
Background- Balance disorder is a common problem in post-TBI patients. In many research's done before treatment is given mainly on motor component to improve balance in TBI patients. In this study we are going to give multidimensional protocol - sensory stimulations along with motor components. Balance is a main component of a patient for his mobility, many therapist concentrate on balance in chronic stage of TBI patients, in this study we are going to give treatment in acute stage of TBI patients which will help them to recover fast. There is lack of research's done on acute post TBI patients with multidimensional exercise program for balance disorder. Indeed this made us to study the effect of multidimensional exercise program on balance disorder in TBI patients. Objectives- To find out the effectiveness of multidimensional exercise program for improving balance control in mild TBI patients. To find out the effectiveness of multidimensional exercise program for improving balance control in moderate TBI patients. To find out the effectiveness of multidimensional exercise program for improving balance control in severe TBI patient. METHOD- In this study total 20 candidates were taken having impaired balance secondary to TBI from hospitals in karad. Their balance were assessed by berg balance scale and community balance and mobility scale. They were divided into mild, moderate and severe TBI. A multidimensional exercise protocol of 6 week was given for improving balance and their balance was checked at 2nd, 4th, and 6th week by same BBS and CBMS score. Later evaluation and interpretation of data was done. Result- The $20.8 \%$ improvement is seen in pre and post treatment BBS score and $8.82 \%$ improvement is seen in pre and post treatment CBMS score in post TBI patients with balance impairment. Conclusion- On the basis of result it can be concluded that multidimensional exercise program is effective for improving balance in TBI patients.
\end{abstract}

Keywords- Traumatic brain injury, Balance, Multidimensional, exercise program, physiotherapy.

\section{Introduction}

Traumatic brain injury is defined as an alteration in brain function caused by an external force. Brain tissue

\footnotetext{
Corresponding author: Dr. Suraj B Kanase, Associate Professor, Department of Neurosciences Physiotherapy, Krishna College of Physiotherapy, Krishna Institute of Medical Sciences Deemed To Be University, Near Dhebewadi Road, Karad, Maharastra, India, 415110

Email id: drsurajkanase7@rediffmail.com

Pranalikakade2810@gmail.com
}

damage can be of two type: primary injury and secondary injury. Primary injury occurs due to direct trauma to the parenchyma. Secondary injury results from cascade of biochemical, cellular, and molecular events that occur due to an initial injury and injury related hypoxia, elevated intracranial pressure and oedema. ${ }^{(1)}$ There is open head injury and close head injury, open head injury is associated with skull fracture and close head injury occurs in isolation without skull fracture. Blood supply of brain may also get indirectly affected due to the injury of face and neck. Most TBI occurs due to road traffic accident (RTA). The severity of brain injury may be categorised as mild, moderate and severe head injury. ${ }^{(2)}$ 
In Mild traumatic brain injury there is minimal damage to the neuroanatomical structures with slight or no permanent impairment. Glasgow coma scale score of more than 13 is defined as mild TBI. In moderate TBI GCS between $8-13$ and the post traumatic amnesia lasting between 1 to 24 hours. Severe TBI is GCS score less than $8 .{ }^{(2)}$

After TBI - state of consciousness, sensorimotor changes, cognitive, emotional and behavioural changes, attention, balance, dizziness and visual dysfunction, spasticity and post traumatic headache. ${ }^{(2)}$

Balance disorder is a common consequence of traumatic brain injury. Balance is a condition in which the centre of mass is within the stability limit and base of support (BOS).Balance is depend on 1) sensory / perceptual systems responsible for the detection of body motion and position.2) motor systems responsible for presenting motor synergies.3) CNS process responsible for integration and action plans. (1) Balance is controlled by cerebellum and basal ganglion in brain, damage to this area may cause loss of control of balance.

To maintain a balance, it is important that central nervous system receives and integrates a somatosensory information, which includes both the tactile and proprioceptive system. The receptors which carry tactile sensations are Merkels cells, Pacinian corpuscles, Meissners corpuscles and Ruffini endings. And they are found in feet and might play an important role in maintaining body stability and balance in upright stance. The proprioceptive receptors are muscle spindle and Golgi tendon organs which carry the sense of joint position and joint motion. The proprioceptive receptors in feet are sensitive to ankle rotation and can give information about balance. ${ }^{(3,4)}$ Stimulation of these receptors may improve balance control in individual with post TBI. The rhythmic auditory stimulation (RAS) also improves the balance and gait of post TBI patients by stimulating brain functions involved in movement, cognition, emotions and sensory perception also RAS helps in execution of movements and normalizing gait parameters. ${ }^{(5,6,7)}$ Balance incorporates two vestibular reflexes- the vestibuloocular reflex (VOR) and the vestibulospinal reflex (VSR). To improve balance after TBI the vestibular balance rehabilitation therapy and visual treatments are commonly used ${ }^{(8)}$

Postural impairment and coordination are common consequences of TBI which may result in instability while performing day today activities like standing, walking, grasping, difficulty while coordinating eyehead movement and problem while focusing on target. The postural and coordination defect result from damage to cerebellum. ${ }^{(9)}$

The motor disability following TBI is a common, motor performance can be affected by damage to various loci in the nervous system which can cause reduction in muscle power and tonus disorder which can result in impaired balance. ${ }^{(6,7)}$ Focal or diffuse spasticity may appear following TBI. Time post injury is an important consideration as spontaneous neurological recovery may continue for 9- 15 months post injury. Motor impairment can also result from prolong immobilization and bed rest during acute period, prolonged immobility can cause effect on multiple body system that impact the motor function the most. Motor rehabilitation is essential in helping the patient re-establishing independence post TBI. ${ }^{(7,8)}$

Impaired confidence in balance skill has been reported to cause depression, fear of falling and limited ability to participate in social activity. ${ }^{(9)}$

Examination of balance in TBI patients is done by using berg balance scale and community balance and mobility scale in ambulatory patients. $(10,11$, 12) Initially many therapist concentrate on the motor component of patient to improve his balance in this study we are going to give multidimensional intervention for balance in acute phase of post TBI patients.

\section{Material and Method}

This was a study to find the effect of multidimensional exercise program for improving balance in TBI patients. The study was carried out in hospitals from Karad. An approval for the study was obtained from the protocol committee and ethical committee of KIMSDU. Individual were approach and those fulfilling the inclusive criteria were selected. The purpose was explained and written inform consent was taken prepared in accordance with the Helsinki Declaration from those who are willingly to participate. Total 20 individuals were taken. The inclusion criteria was Acute TBI individuals, candidates with mild, moderate and severe TBI, Both male and females, Age- above 18 years and candidates with injury related balance disorder. The exclusion criteria was individual who are unable to follow simple commands, Intact balance after injury, unconscious patients, unstable vitals and candidates with any 
fracture of spine, lower and upper limb. Their balance was assessed by berg balance scale and community balance and mobility scale. Their tone was assessed by modified Ashworth's scale and stage of TBI were seen by initial GCS score. Then the candidates were given multidimensional exercises such as sensory training, bed mobility exercises - segmental rolling, pelvic bridging and heel slides. Active or active assisted ROM exercises to prevent contractures. Tone management by stretching and icing, proprioception training by joint compression, auditory clues by rhythmic auditory stimulation, visual feedback, vestibular rehabilitation exercises, balance and coordination training. This was given for 4 days per week for 6 weeks up to 45-60 minutes per session including rest time. The time of session increased as the week progresses, initially it took 30- 35 minutes per session and later progressed to 45- 60 minutes per session on 6 th week. ${ }^{(13)}$ The balance was checked at 2 nd, 4 th and 6th week by same berg balance scale and community balance and mobility scale to know the progression of candidates. Also the candidates were divided into mild, moderate and severe TBI. Later evaluation and interpretation was done using statistical analysis.

\section{Statistical Analysis}

The paired $\mathrm{T}$ test and one-way ANOVA test were used for analysis of data. Statistical analysis of the recorded data was done by using the software SPSS version 20. The $\mathrm{p}$ value is less than 0.0001 which is extremely significant for pre and post treatment BBS score and CBMS score.

\section{Findings}

The significant difference is seen in pre and post score of berg balance scale and community balance and mobility scale in post TBI patients with impaired balance after multidimensional exercise program of 6 week. The $20.8 \%$ improvement is seen in pre and post treatment BBS score and $8.82 \%$ improvement is seen in pre and post treatment CBMS score in post TBI patients with balance impairment.(p value $=<0.0001$ ). While the mild TBI candidates showed $27.38 \%$ more progression in BBS score and $18.38 \%$ more progression in CBMS score as compared to severe TBI candidates. $(p$ value $=$ $>0.05)$

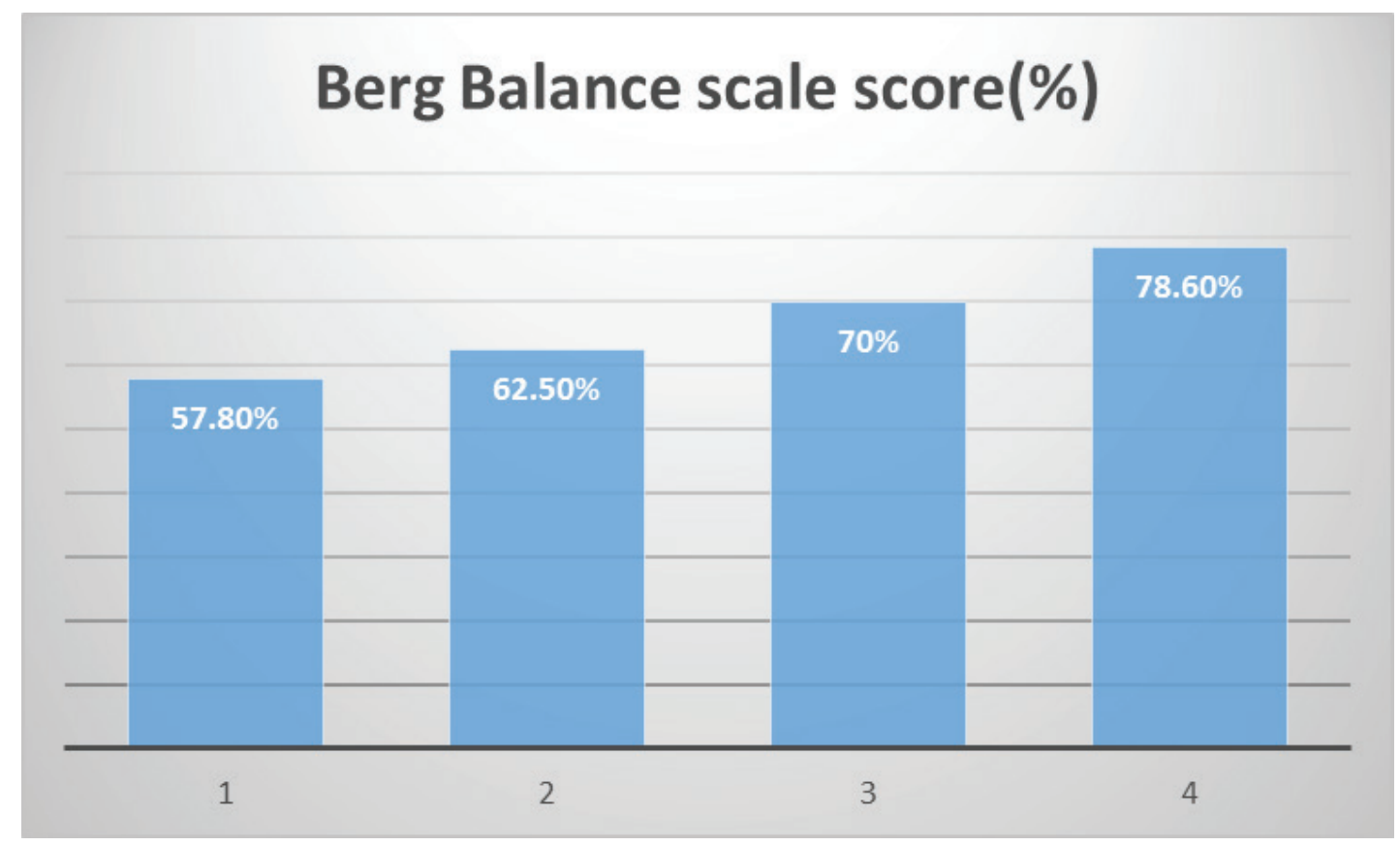

Graph No.1- Comparison of Pre And Post Treatment Berg Balance Scale Score.

INTERPRETATION- The above graph shows the comparison of berg balance scale score of pre-treatment, post treatment 2 weeks, post 4 weeks and post 6 weeks score in percentage. It shows significant improvement in score. 1) Pre-treatment score is $57.80 \%, 2$ ) Post treatment 2 weeks score is $62.50 \%, 3$ ) Post treatment 4 week score is $70 \%$ and 4) Post treatment 6 week score is $78.60 \%$. 


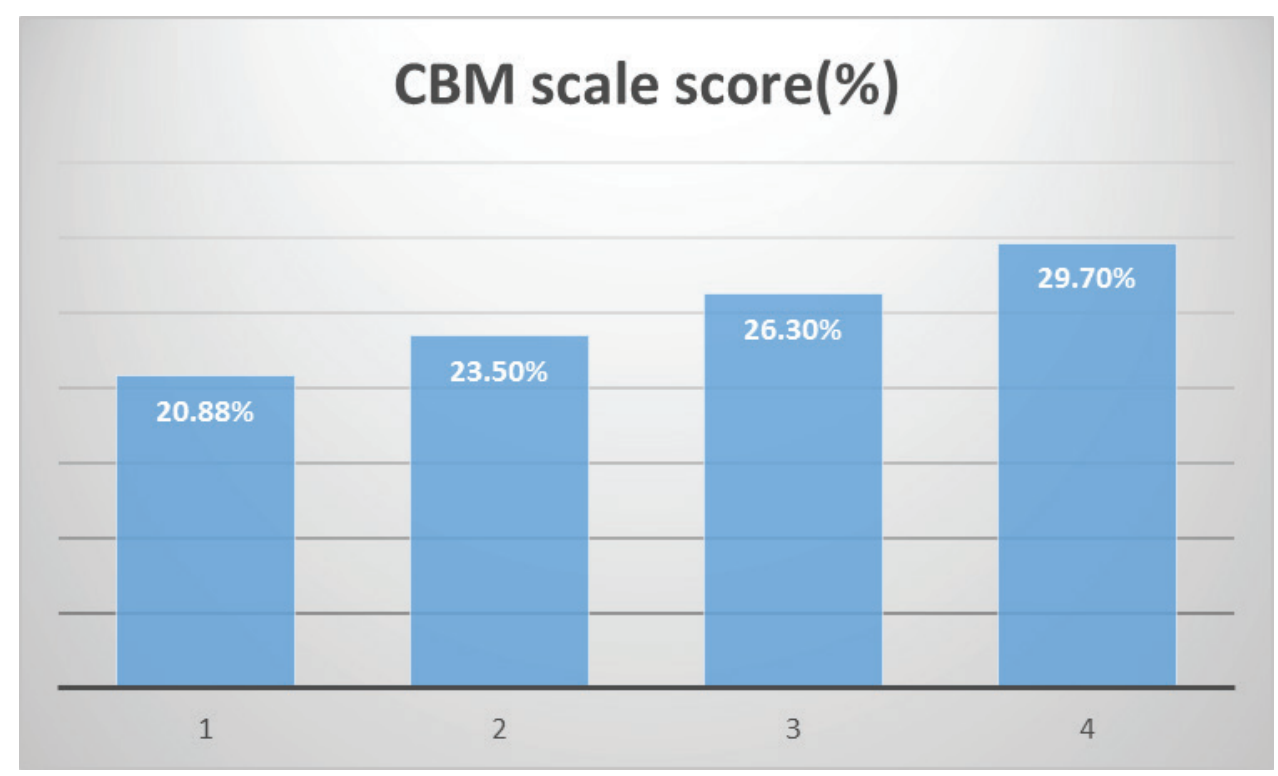

\section{GRAPH NO.2- COMPARISON OF PRE AND POST TREATMENT COMMUNITY BALANCE AND MOBILITY SCALE SCORE.}

INTERPRETATION- The above graph shows the comparison of community balance and mobility scale score of pre-treatment, post treatment 2 weeks, post 4 weeks and post 6 weeks score in percentage. It shows significant improvement in score. 1) Pre-treatment score is $20.88 \%$, 2) Post treatment 2 weeks score is $23.50 \%$, 3) Post treatment 4 week score is $26.30 \%$ and 4 ) Post treatment 6 week score is $29.70 \%$.

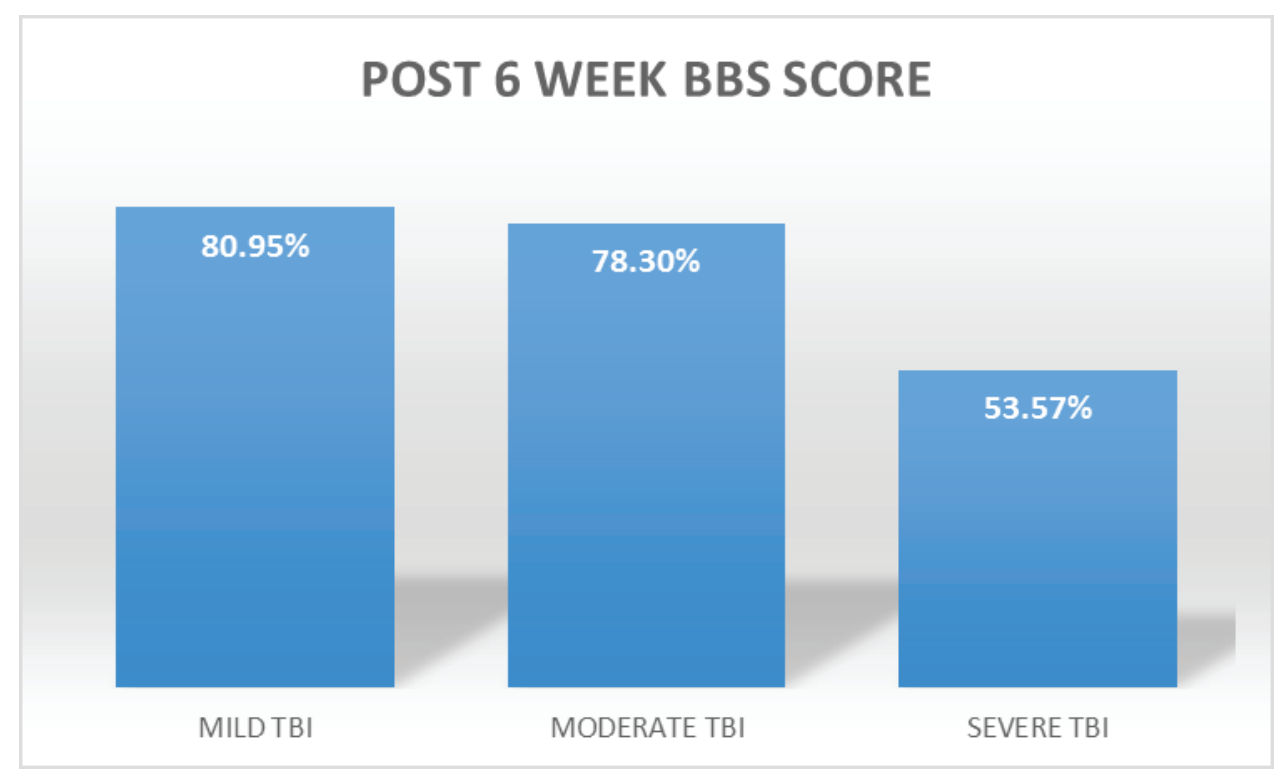

GRAPH NO.3- COMPARISON OF MILD, MODERATE AND SEVERE TBI PATIENTS POST 6 WEEK BBS SCORE

INTERPRETATION- The above graph shows the comparison of BBS score post 6 week of treatment in mild, moderate and severe TBI patients in percentage. More improvement is seen in mild TBI patients then moderately and severed injured patients. 


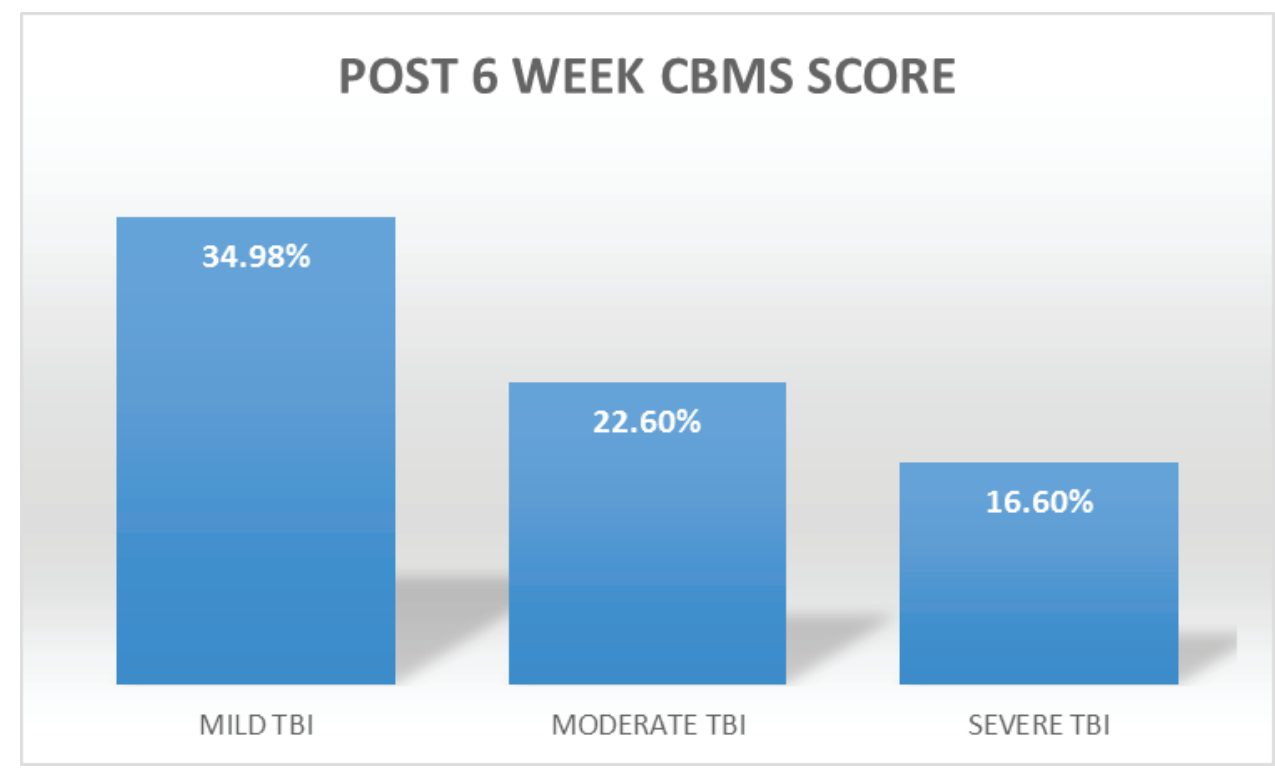

GRAPH NO. 4- COMPARISON OF MILD, MODERATE AND SEVERE TBI PATIENTS POST 6 WEEK CBMS SCORE.

INTERPRETATION- The above graph shows the comparison of CBMS score post 6 week of treatment in mild, moderate and severe TBI patients in percentage. More improvement is seen in mild TBI patients then moderately and severed injured patients.

\section{Discussion}

The purpose of this study was to find out the effectiveness of multidimensional exercise program for improving balance in TBI patients. About $30 \%$ of patients report impaired balance after TBI. ${ }^{(14)}$ In many research's done before treatment was given mainly on motor component to improve balance in TBI patients. Many currently practicing physiotherapist mainly concentrate only on motor components of patients. (7) Multicomponent exercise program have been found to be effective in managing various impairments following stroke $(15,16)$. In this study we have given multidimensional protocol like sensory stimulations like tactile, auditory and visual feedback along with motor components like strengthening of trunk muscles, management of muscle tone, balance and coordination training and also prevention of contractures.

Our study included total 20 post TBI candidates with impaired balance. From which $60 \%$ candidates were of mild TBI, $35 \%$ were of moderate TBI and $5 \%$ candidate of severe TBI. In which $40 \%$ were females and $60 \%$ were males with TBI.

Balance disorder is a common problem in post-TBI patients. This problem may persist for life time after TBI which can affect the Patients daily activities and his mobility. For this early management of patients in its acute stage plays an important role.(17) In this study we took a candidates of acute stage from which $30 \%$ of them was having impairment in only dynamic balance and $70 \%$ were having impairment in both static and dynamic balance. The candidates having impairment in only dynamic balance was also given static balance exercises training to gain a confident in ambulation.

Motor management alone with sensory integration have a more effect in improving balance in post TBI patients. Vestibular apparatus has also been identified as sensory organ that controls sensations of balance and equilibrium in individual. In this study we found that candidates also had impairment in eye-head coordination movements which was improved by vestibular exercises which help in stabilizing gaze and eventually to improve the balance. The vestibular- ocular reflex supports stabilising vision while head is moving. (18) Vestibular rehabilitation is also effective in managing vertigo and dizziness which are commonly found after TBI. ${ }^{(19)}$

Evidence shows that auditory and motor system has high connectivity, hence many research have shown that RAS to be more effective than other sensory cues in rehabilitation. ${ }^{(8)}$ Also RAS helps in motor relearning and neuronal plasticity. ${ }^{(20)}$

Stimulation to proprioceptors and somatosensory receptors in ankle and foot are also known to improve 
balance. ${ }^{(3,4)}$

Motor disturbances following TBI includes reduction of power, tonus disorder (spasticity) and motor control disorganisation. Reduction of spasticity helps in improving ROM and functional activities. The static balance is improved by decreasing muscle tone of neck and upper trunk. Thus improving balance of individuals. $(21,22)$

The strength training for core muscles and lower limb also reported to improved patients balance and confidence in daily activities.

In the study made by Vishwajeet Trivedi, et al. showed the satisfactory effect of strength and balance intervention in improving balance, gait pattern and function independency in TBI Patient. Our research also support this study as in our study we have used strength and balance intervention for improving balance in post TBI. ${ }^{(23)}$

Limitation of this study was that the study is done with small sample size and in limited geographical area.

\section{Conclusion}

On the basis of the results it is concluded that multidimensional exercise program is effective in improving balance in Traumatic brain injury patients. Multidimensional approach improves the balance in various grades of head injury.

Conflicts of Interest: There is no conflict of interest in this study.

Source of Funding: The study was funded by Krishna institute of medical sciences deemed to be university, karad.

Ethical Clearance: This study has undergone ethical clearance through the university level ethical committee. Protocol number 074/2019-2020.

\section{References}

1. Geoge D. Fulk, Traumatic Brain Injury. In: Susan B. O'Sullivan, Thomas J. Schmitz, George D. Fulk editor. Physical rehabilitation, 6th ed. New Delhi: Jaypee brothers medical publishers. 2014. p. 85963.

2. Glady Samuel Raj, Head injury. In: Glady Samuel Raj editor. Physiotherapy in neuro-conditions, 1st ed. New Delhi: Jaypee brothers medical publishers.
2006. p. 78-96.

3. HJJ Kars, Juha M Hijmans, Jan HB Geertzen, Wiebren Zijlstra. The effect of reduced somatosensation on standing balance: a systemic review. journal of diabetes science and technology 2009;3(4):931-43

4. Ming-Hsia Hu, Marjorie Hines Woollacott. Multisensory training of standing balance in older adukts: Postural stability and one- leg stand balance. Journal of gerontology 1994;49(2):M52-M61

5. Joke Bradt, Wendy L Magee, Cheryl Dileo, Barbara L Wheeler, Emer McGilloway. Music therapy for acquired brain injury. the Cochrane collaboration 2010;(7):1-42

6. Corene P. Hurt, Ruth R. Rice, Gerald C. McIntosh, Michael H. Thaut. Rhythmic Auditory Stimulation in Gait Training for Patients with Traumatic Brain Injury. Journal of Music Therapy 1998;35(4): 228 241

7. OferKeren, Jacqueline Reznik andZeev Groswasser. Combined motor disturbances following severe traumatic brain injury: an integrative long- term treatment approach. Brain Injury 2001;15(7):63338

8. Michael H. Thaut, Mutsumi Abiru. Rhythmic Auditory Stimulation in Rehabilitation of Movement Disorders: A Review Of Current Research. An Interdisciplinary Journal 2101;27(4)

9. Harold A., John Shelley-Tremblay, Steven Larson. Vestibular function, sensory integration, and balance anomalies: A brief literature review. Optom Vis Dev 2007;38(1):13-17

10. Ksenia I, Ludmila A, Chernikova and Jan Perkins. Physical therapy for correcting postural and coordination deficits in patients with mild to moderate TBI. Physiother theory pract,2014;31:1-7

11. Newstead, Ann H., Hinman, Martha R., Tomberlin, Jo Ann. Reliability of the Berg Balance Scale and Balance Master Limits of Stability Tests for Individuals with Brain Injury. Journal of Neurologic Physical Therapy 2005;29(1):18-23

12. J A Howe, E L Inness, A Venturini, J I Williams. The Community Balance and Mobility Scale-a balance measure for individuals with traumatic brain injury.Clinical rehabilitation 2006;10(20): 885-95

13. Bara A. Alsalaheen, et al. Exercise prescription patterns in patients treated with vestibular 
rehabilitation after concussion. Physiother. Res. Int 2013;18:100-08

14. Alexander C. H. Geurts, Gerardus M. Ribbers, Johannes A. Knoop, Jacquesvan Limbeek: Identification of static and dynamic postural instability following traumatic brain injury. Archives of Physical Medicine and Rehabilitation 1996;77(7): 639-44.

15. Vispute AR, Kanase SB. Effect of electrical stimulation, hot moist pack and exercises on shoulder hand syndrome in stroke patients. Global journal of research analysis. 2018.

16. Sawant VS, Kanase SB. Effect of conventional therapy and positioning with isometric shoulder exercises on functional shoulder subluxation in post stroke survivors. Indian journal of public health research \& development. 2019;10(8):536-40

17. McCrea MA, Nelson, L.D., Guskiewicz: Diagnosis and management of acute concussion.Med. Rehabil. Cli 2017;28: 271-86

18. Neil T. Shepard, Steven A. Teljan, Michael SmithWheelock, Anil Raj. Vestibular and balance rehabilitation therapy. Annals of otology, rhinology and laryngology 1993;102(3):198-205
19. James M. Gurley, Bryan D. Hujsak, Jennifer L. Kelly: Vestibular rehabilitation following mild traumatic brain injury. Neuro Rehabilitation 2013;32:519-28

20. Rebecca S. Schaefer. Auditory rhythmic cueing in movement rehabilitation: finding and possible mechanisms. PHILOSOPHICAL transactions of Royal Society B.2014;1-9

21. Pattuwage, et al: Management of Spasticity in Moderate and Severe Traumatic Brain Injury Evaluation of Clinical Practice Guidelines.The Journal of Head Trauma Rehabilitation 2017;32 (2): E1-E12

22. M.J.Killington, S.F.H.Mackintosh, M. Ayres. An isokinetic muscle strengthening program for adults with acquired brain injury leads to meaningful improvements in physical function.Brain injury 2010;24:970-77

23. Vishwajeet Trivedi, Kunal Thakur, Rakesh Choudhary, Pallavi sahay: Effect of strength and balance intervention in improving balance, gait and function in traumatic brain injury: A case report. International journal of health sciences and research 2014;4(6):259-66. 